Revista de Psicología Vol. 35 (2), 2017 (ISSN 0254-9247)

\title{
Factores sociodemográficos explicativos del guion de base segura materno ${ }^{1}$
}

\author{
Magaly Nóblega ${ }^{2}$, Pierina Traverso ${ }^{3}$, Andrea Ugarte ${ }^{4}$, Luciana Caballero ${ }^{5}$ \\ Pontificia Universidad Católica del Perú
}

Este estudio evalúa la capacidad predictiva de variables sociodemográficas de la madre sobre sus guiones de base segura. Participaron 83 madres de 15 a 45 años de edad $(M=24.72$, $D E=8.70)$. El nivel de base segura materno fue evaluado a través de las Narrativas de Apego Adulto (Waters \& Waters, 2006). Los resultados muestran que las participantes no cuentan con un adecuado nivel de base segura. Se encontró que la interacción entre la edad y el nivel de instrucción materno explica en parte el nivel de base segura de las madres $\left(R^{2}=.19\right)$. Se postula el rol protector del nivel de instrucción para que las madres mayores, tengan un mayor nivel de base segura en sus guiones.

Palabras clave: guión de base segura, edad, nivel educativo

1 Esta investigación incluye los datos de dos estudios, uno financiado por la Dirección de Gestión de la Investigación de la PUCP, a través de la subvención DGI 70243.0022. El otro fue co-financiado por el Research Council de la KU Leuven, Bélgica y por la Dirección de Gestión de la Investigación de la PUCP.

2 Doctora en Psicología, docente del Departamento de Psicología de Pontificia Universidad Católica del Perú. Miembro del Grupo de Investigación Relaciones Vinculares y Desarrollo Socioemocional. Dirección postal: Av. Ramírez Gastón 266 - 302. Lima 18. Contacto: mnoblega@pucp.pe

3 Doctora en Psicología, docente del Departamento de Psicología de Pontificia Universidad Católica del Perú. Dirección Postal: Av. Las Acacias 196 - 601. Lima 18. Contacto: ptraver@ pucp.pe

4 Licenciada en Psicología Clínica, docente del Departamento de Psicología de Pontificia Universidad Católica del Perú. Miembro del Grupo de Investigación Relaciones Vinculares y Desarrollo Socioemocional. Dirección postal: Av. Roca Bolońa 251 - 603. Lima 18. Contacto: andrea.ugarte@pucp.pe

5 Licenciada en Psicología Clínica, docente del Departamento de Psicología de Pontificia Universidad Católica del Perú, Lima, Perú. Dirección postal: Calle Severini 114. Lima 41. Contacto:mgcaballero@pucp.pe 


\section{Maternal Secure Base Scripts' socio demographic predictive variables}

This study evaluates the predictive capacity of sociodemographic variables of the mother over the level of maternal secure base scripts. The participants were 83 mothers from 15 to 45 years old $(M=24.72, S D=8.70)$. The level of maternal secure base was evaluated through the Narratives of Adult Attachment (Waters \& Waters, 2006). The results show that the participants do not have an adequate level of secure base script. It was found that interaction between the age of the mother and the educational attainment level, partly explains the level of the maternal secure base script $\left(\mathrm{R}^{2}=.19\right)$. The protector role of the level of educational attainment for older mothers to have a higher level of secure base scripts is proposed.

Keywords: secure base script, age, educational attainment level

\section{Fatores sócio-demográficos explicativos do script de base segura materno}

Esta pesquisa avalia a capacidade preditiva das variáveis sócio-demográficas da mãe, a partir de seu script de base segura. Participaram 83 máes com idades entre 15 e 45 anos $(M=24.72, D E=8.70)$. O nível de script de base segura materno foi avaliado através das Narrativas de Apego Adulto (Waters \& Waters, 2006). Os resultados mostram que as participantes não apresentam um nível adequado de base segura. Encontrou-se que a interação entre idade e grau de instrução da mãe explica, em parte, o nível de base segura das mães $\left(\mathrm{R}^{2}=.19\right)$. Propóe-se que um grau de instruçáo maior constituiria um papel protetor nas máes mais velhas, para elas apresentarem maior nível de base segura nos seus scripts.

Palavras-chave: script de base segura, idade, educação 
La calidad de las experiencias con el cuidador durante la infancia y la base segura que este haya provisto, son internalizadas por la persona en lo que Bowlby (1969) denominó modelos operativos internos (MOI). Estos son descritos como representaciones de apego, estructuras que organizan las creencias acerca de sí mismo y los otros (Bowlby, 1969; Marrone, 2001).

Bowlby sugirió que las representaciones de apego adultas son reflejadas en todas las relaciones cercanas del adulto como son las relaciones de pareja (Hazan \& Shaver, 1987; Simpson, Collins, Tran \& Haydon, 2007) y/o en el ejercicio de la parentalidad (Grossmann, Grossman \& Kindler, 2005; Main, 2000; Rholes, Simpson \& Friedman, 2006). De esta manera, reflejan el grado de seguridad en las relaciones cercanas $\mathrm{y}$ tienen un rol importante en el modo en que un individuo se siente respecto a los demás; en cómo espera ser tratado; y en cómo trata a los demás (Bretherton, 1990; Bretherton \& Munholland, 2008; Main, Kaplan \& Cassidy, 1985; Morales \& Santelices, 2007; Pinedo \& Santelices, 2006; Waters \& Rodrigues, 2001).

En el campo del apego adulto hay diferentes perspectivas para acceder al estudio de estas representaciones (Steele et al., 2014). Por un lado, es posible analizar la narrativa de la persona como en la Entrevista del Apego Adulto (AAI, por sus siglas en inglés) o determinar los niveles de ansiedad y evitación de un individuo como dimensiones de su estilo de apego (Mikulincer \& Shaver, 2007) o evaluar la inclusión del fenómeno de base segura en sus guiones (Waters \& Rodrigues-Doolabh, 1998; Waters et al., 2015). Estos últimos pueden ser considerados una manifestación central de las representaciones de apego que se activan ante una situación específica (Bakermans-Kranenberg, 2006).

Desde esta última perspectiva, los "guiones de base segura" (secure base scripts) implican que las experiencias que tiene una persona con su figura de apego son interiorizadas en secuencias de eventos en la 
memoria (Bretherton, Ridgeway \& Cassidy, 1990; Waters \& Rodrigues-Doolabh, 1998; Waters \& Waters, 2006) y se organizan a modo de un guion (Waters \&Waters, 2006).

Bajo esta premisa, Waters y Rodrigues-Doolabh (2001) propusieron que en las narrativas discursivas de las personas existiría un prototipo de guion de base segura en donde 1) la figura de apego incentiva la exploración del individuo, 2) esta figura está disponible para el individuo, 3) el individuo se topa con un obstáculo, 4) el individuo se refugia en la figura de apego o esta se acerca al individuo, 5) se resuelve el obstáculo, 6) la proximidad de la figura de apego calma al individuo, 7) el individuo retorna a la actividad.

De este modo, las personas que durante su infancia han tenido interacciones con un cuidador que funcionó como base segura, durante la adultez accederán a dicho guion para relacionarse con otros (Bakermans-Kranenburg, 2006; Vaughn et al., 2006; Vaughn et al., 2007; Waters \& Waters, 2006), siendo estos generalizables a diferentes situaciones, pero al mismo tiempo flexibles y específicos a cada situación (Fivush, 2006). Aquellos que se acerquen más al guion prototípico y puedan resolver las situaciones adversas que se presenten tendrán mayor nivel de base segura (Waters, Rodrigues \& Ridgeway, 1998).

Si bien estos guiones de base segura tienden a ser estables a lo largo del tiempo (Allen, Boykin, Kuperminc \& Jodl, 2004; Fivush, 2006; Vaughn et al., 2006; Waters \& Waters, 2006; Waters et al., 2006), las nuevas experiencias se van integrando a las estructuras existentes (Allen et al., 2004; Crowell \& Waters, 2005; Hamilton, 2000; Schoenmaker et al., 2015; Weinfield, Sroufe \& Egeland, 2000). Estas experiencias pueden ser eventos disruptivos como la pérdida de una persona significativa (Hamilton, 2000), o eventos positivos como el establecimiento de una adecuada relación de pareja (Crowell, Treboux \& Waters, 2002; Wais \& Treboux, 2003).

La existencia de este prototipo del guion de base segura ha sido empíricamente comprobada en diversos países y contextos (Coppola, Vaughn, Cassibba \& Costantini, 2006; Rodrigues-Doolabh, Zevallos, Turan \& Green, 2003; Verissimo \& Salvaterra, 2006; Waters \& Waters, 2006). 
De la misma manera, se ha comprobado la relación entre el nivel de base segura de los guiones con otras medidas de las representaciones de apego en el adulto (Coppola et al., 2006; Dykas et al., 2006; Waters \& Rodrigues, 2001; Steele et al., 2014; Waters, Brockmeyers \& Crowell, 2013).

Ahora bien, como ya se expresó los guiones de base segura se pueden activar en la relación con los hijos (Bowlby, 1969; Main, 2000; Rholes, Simpson \& Friedman, 2006). Específicamente, se postula que dentro de la interacción madre-niño, una madre con mayores niveles de base segura en sus guiones podrá adoptar con mayor facilidad una respuesta sensible hacia su hijo (Coppola et al., 2006) y por tanto permitirá que el niño establezca un apego seguro con ella (Monteiro, Veríssimo, Vaughn, Santos \& Bost, 2008; Raval et al., 2001; Tini, Concordan, Rodrigues-Doolabh \& Waters, 2003; van Ijzderdoorn, 1995; von der Lippe, Eilertsen, Hartmann \& Killèn, 2010; Wong et al. 2011), contribuyendo a su vez a la construcción de los guiones de base segura del niño/a (Guttmann-Steinmetz, Elliot, Steiner \& Waters, 2003).

Dada la relativa flexibilidad de los guiones de base segura, además de las relaciones tempranas, otros factores personales de la madre (edad, nivel de instrucción y número de hijos), así como de su contexto micro y macro social (por ejemplo nivel socioeconómico, entre otros) pueden explicar la existencia del fenómeno de la base segura en sus guiones. Al respecto, los estudios para explorar las diferencias en el guion de base segura a partir de las características sociodemográficas maternas son escasos, dado que las investigaciones se realizan con muestras reducidas y muy homogéneas en dichas variables (Anafarta, 2007; Verissimo \& Salvarreta, 2006).

Dentro de los pocos estudios encontrados, Huth-Bocks (2014) resalta que en su muestra de alto riesgo, en donde las participantes habían tenido historias de violencia, el ingreso familiar y la edad de la madre resultan esenciales para entender las asociaciones entre los guiones y la conducta materna. 
Respecto a la edad de la madre, es posible que los guiones sean distintos entre las madres adolescentes y las adultas (Sánchez \& Hidalgo, 2002), ya que a mayor edad, más experiencias y con esta mayor información sobre el mundo y específicamente sobre las relaciones (Lamb, Thompson, Gardner \& Charnov, 1985; Sroufe, 2005). Además, Allen y Land (1999) consideran que durante la adolescencia se integran las representaciones de apego de las distintas figuras cuidadoras gracias al desarrollo cognitivo y a la diferenciación que el adolescente adquiere respecto a los demás. Sin embargo, se considera que la exposición de los adolescentes a las demandas de los padres y la dificultad para obtener soporte de los mismos, podrían disminuir los niveles de seguridad de sus representaciones (Allen, 2008). Si a este proceso le sumamos la experiencia de maternidad, las adolescentes podrían presentar menor nivel de base segura en los guiones, al tener que lidiar también con una nueva relación que debe ser integrada a las anteriores (Sánchez \& Hidalgo, 2002).

Además de la edad, un aspecto a considerar y que interactúa con las experiencias que se van teniendo es el grado de instrucción de la persona. Dado que un mayor nivel de instrucción se relaciona con mayores habilidades lingüísticas, es importante considerar que toda actividad discursiva, como las narrativas, podría verse influida por esta variable (Benziesle \& Mychasiuk, 2009; López-Higes, Rubio-Valdehita, Prados $\&$ Galindo, 2013). Por otro lado, Vaughn et al. (2007) encuentra que los guiones de las madres colombianas estaban asociados al grado de instrucción.

En este punto, se debe considerar que el nivel de instrucción y el nivel socioeconómico son variables que interactúan dado que en los niveles más bajos, por lo general, las personas tienen un menor grado de instrucción debido a que las adversidades del entorno no han permitido que los estudios sean una prioridad (Pelchat, Bisson, Bois \& Saucier, 2003). En el caso del embarazo adolescente, por ejemplo, las madres tienden a dejar sus estudios a partir del embarazo (Chalasani, Kelly, Mensch \& Soler-Hampejsek, 2013). 
Asimismo, cabe subrayar que el nivel socioeconómico bajo trae consigo una serie de experiencias negativas que son internalizadas y que pueden afectar al desenvolvimiento de la persona, especialmente si hablamos de contextos de pobreza (Adler et al., 1994; De Falco et al., 2014). La pobreza conlleva a la existencia de eventos estresantes y disruptivos que pueden afectar las condiciones psicológicas de los individuos y específicamente la capacidad para que una madre se vincule sensiblemente con su hijo(a) (Belsky, 1984; van Ijzerdoorn \& Bakermans, 1996). Así, los adultos que han vivido en estas condiciones siendo nińos, tenderían a tener guiones menos seguros (Huth-Bocks et al., 2014).

Finalmente, los diferentes eventos que la maternidad supone proveen información y complejizan las experiencias de los guiones de base segura (Fivush, 2006), lo cual también se irá incrementando de acuerdo a la cantidad de hijos y probablemente a sus respectivas edades. En relación al número de hijos, se reporta que las madres con mayor experiencia en la maternidad sienten que cuentan con mayor capacidad para brindar un cuidado adecuado a sus hijos comparadas con las madres primerizas (Kapp, 1998), quienes por el contrario, tienden a tener sentimientos de inseguridad en relación a su rol maternal (Coleman \& Karraker, 2000). Esta mayor autoconfianza y la experiencia previa de maternidad conllevan a que la madre experimente emociones positivas, lo cual podría influir de manera positiva en su guion de base segura.

A partir de lo expuesto, se evidencia una escasez de estudios en relación a las variables sociodemográficas asociadas al nivel de base segura de los guiones, por lo que es importante el estudio de estas variables sociodemográficas y cómo estas interactúan o no, en la coherencia, consistencia y calidad de los guiones de base segura. Por ello, esta investigación evaluó el valor explicativo de factores sociodemográficos maternos — edad, grado de instrucción, número de hijos y nivel socioeconómico- sobre el nivel de base segura de los guiones de un grupo de madres. Este estudio es relevante en un contexto en el que la maternidad es vivenciada en diversas condiciones personales y socioculturales. 


\section{Método}

\section{Participantes}

Las participantes fueron 83 madres que tenían por lo menos un hijo menor de 6 años. La edad promedio de las madres fue de 24.72 $(D E=8.7), 25$ de ellas eran adolescentes con edades que oscilaban entre 15 y 19 años, 35 eran madres jóvenes con edades entre 20 y 30 años y 23 eran madres mayores con edades que fluctuaban entre los 31 y los 45 ańos.

En cuanto a su nivel de instrucción, el 56.6\% había culminado sus estudios secundarios y el $43.4 \%$ de las madres tenían estudios técnicos. Además, con respecto a la ocupación, el $36.1 \%$ de las madres trabajaban o estudiaban mientras que el $63.9 \%$ restante se dedicaban exclusivamente a las labores del hogar. En referencia a su situación conyugal, el $59 \%$ de las madres vivían con el padre biológico del hijo.

El número de hijos de las madres fluctuaba entre 1 y $3(M o=1$, $D E=.66)$, siendo 65 las madres que tenían un sólo hijo y 18 las que tenían más de 1 hijo. En relación al género del hijo menor, 45 eran niños y 38 niñas. La edad del hijo menor varió entre 2 y 72 meses siendo en promedio $18.63(D E=23.77)$.

Las madres pertenecían a dos niveles socioeconómicos (NSE) bajo y medio, definidos a partir de la zona de residencia y delimitados por los dos estudios de los cuales provienen las participantes de esta investigación. El primero de ellos incluyó a madres adolescentes y jóvenes de NSE bajo que tenían un único hijo menor a 5 meses, mientras que en el segundo estudio participaron madres no adolescentes con por lo menos un hijo menor de 6 años.

En el primer estudio, las madres fueron seleccionadas del grupo de mujeres que asistían a un centro de salud para el control médico de su hijo, a ellas se les explicaba la naturaleza del estudio solicitándoles su participación en el mismo. En el segundo estudio, las madres y sus hijos fueron seleccionados a través de la institución educativa a la que asistían sus hijos. A las madres que respondieron positivamente a la 
invitación escrita enviada, se les explicó la naturaleza de la investigación solicitándoles su participación voluntaria.

En ambos grupos, las madres que aceptaron participar voluntariamente, firmaron un consentimiento informado en señal de su aceptación para participar en el estudio. En este se explicaba la naturaleza de su participación, los beneficios, así como los potenciales riesgos, además se aseguraba la confidencialidad de los datos recogidos y la exclusividad de su uso para fines de la investigación.

\section{Medición}

El guion de base segura se evaluó a partir de la construcción de historias utilizando como estímulo un grupo de palabras predefinidas que constituyen las Narrativas de Apego Adulto (Rodrigues-Doolabh, Wais, Zevallos \& Rodrígues, 2001).

La prueba consta de 4 grupos de palabras (prompts) planteadas para activar el guion de base segura de la madre en diferentes escenarios: dos de la interacción madre-hijo (La mañana del bebé y La visita al doctor) y las otras dos de interacciones entre adultos, las cuales pueden ser interpretadas como una relación de pareja (Ferias y fiestas ${ }^{6}$ y El accidente). Además, la prueba tiene otras dos historias de control (Un paseo al parque y Una tarde de compras) las cuales no se consideran en la calificación del guion de base segura.

La historia dada por la madre es evaluada de acuerdo al guion de base segura que contiene la historia. Esta historia se califica en una escala de 7 puntos en la que a mayor puntuación, mayor nivel de base segura en el guion. En general, las historias con un mayor puntaje son aquellas en las que la base segura (madre o pareja) ayuda al personaje de la historia a lidiar con la situación estresante y a retornar a la normalidad. En estas historias, hay además, un alto grado de atención al estado psicológico del otro y la base segura se muestra responsiva a dicho estado psicológico. En cambio, las historias con menores puntajes son aquellas en las que no se identifica una persona que pueda actuar como base segura

6 La historia original se titula El campamento. 
ante la dificultad evidenciada, no hay un retorno a la normalidad, ni una solución al problema. En algunos casos estas historias presentan contenido extraño como comentarios inconsistentes con el guion de base segura o son historias que presentan una serie incoherente de eventos (Waters \& Waters, 2006). Además, es importante mencionar que las historias con calificaciones inferiores a 4 pueden clasificarse como guiones con bajo nivel de base segura o inseguros.

Para cada madre se obtiene como puntuación el promedio de las 4 historias (La mañana del bebé, Visita al doctor, Ferias y fiestas, y El accidente), lo que constituye el nivel de base segura. Asimismo, se obtiene el promedio de las dos historias de interacción madre-bebe y el de las dos historias de interacciones adultas.

Como una evidencia de la validez de constructo, Waters y Waters (2006) demostraron que las puntuaciones de las historias de interacción madre-bebe, así como las puntuaciones de las interacciones entre adultos se encuentran altamente correlacionadas $(.80<r<.90)$ y que los promedios de ambas puntuaciones se encuentran asociados $(r>.50)$. En este estudio, las puntuaciones de las narrativas de las historias de la interacción madre-bebe y las de adultos estuvieron significativamente asociadas (.58, y .45 respectivamente); asimismo las puntuaciones promedio de estas, también correlacionaron significativamente (.52).

La prueba tiene evidencias de validez convergente con el índice de coherencia de la Adult Attachment Interview (Waters \& RodriguesDoolabh, 2001) y de validez predictiva con la sensibilidad materna (Coppola et al., 2006), así como con la clasificación del apego de los hijos (Tini, Corcoran, Rodrigues-Doolabh \& Waters, 2003) y también con la conducta de base segura de hijos biológicos (Bost, et al., 2006) y de hijos adoptados (Verissimo \& Salvaterra, 2006).

Por otro lado, la universalidad del guion de base segura ha recibido evidencias en estudios en los que participaron mujeres de distintos contextos: Estados Unidos, Suiza, Italia, Turquía, Zimbawe, Emiratos Árabes Unidos y Perú (Coppola et al., 2006; Rodrigues-Doolabh, Zevallos, Turan \& Green, 2003; Rodrigues-Doolabh et al., 2001). 
Con relación a la confiabilidad del instrumento en el presente estudio, dos evaluadores previamente entrenados en la aplicación y calificación del instrumento, calificaron de manera independiente las narrativas. La confiabilidad interevaluador obtenida a través de la correlación intraclase fue de .94 para la puntuación global, de .96 y .84 para las narrativas de interacciones madre-hijo y de adultos respectivamente y entre .88 y .96 para cada una de las narrativas. Con la finalidad de mejorar la evaluación de las narrativas de las participantes, los calificadores discutieron las puntuaciones en las que había una diferencia de 1 punto o más entre las puntuaciones independientemente asignadas por cada uno de ellos. Estas historias constituyeron el 7.26\% de las calificaciones. De esta manera, la puntuación del nivel de base segura de cada madre fue el promedio de las puntuaciones de ambos calificadores cuando había una discrepancia menor a 1 en las calificaciones independientes; mientras que en los casos en los cuales la discrepancia era mayor a 1, la puntuación otorgada fue el consenso al que llegan ambos calificadores luego de la discusión.

Adicionalmente, la confiabilidad por consistencia interna para las 4 narrativas fue de .72. En el caso de las dos narrativas de escenarios de interacción madre-bebe fue de .73 , mientras que para las dos narrativas que planteaban escenarios de interacción entre adultos fue de .59 .

\section{Procedimiento}

En el caso del primer grupo de participantes de NSE bajo, las madres fueron evaluadas como parte de un estudio mayor en el que comparaban las representaciones mentales e interacción madre-bebe entre madres adolescentes y madres adultas, en este grupo las narrativas maternas fueron evaluadas en la segunda y última reunión que se tuvo con las madres. En el segundo grupo de NSE medio, las madres participaron de un estudio en el que se evaluaron los aspectos comportamentales y representacionales del apego del niño y de la conducta materna, en este grupo las narrativas fueron evaluadas en la primera reunión que se tuvo con las madres. 


\section{Análisis de datos}

Los datos se procesaron utilizando el paquete estadístico SPSS versión 19 (IBM Inc., 2010) y para el cálculo de la potencia post hoc de las pruebas estadísticas se utilizó el programa G Power versión 3.1.3 (Faul, Erdfelder, Buchner \& Lang, 2009). Dado que no se encontraron datos perdidos, no se realizaron los análisis correspondientes.

Una vez realizados los análisis exploratorios de la data y previa verificación de la ausencia de datos perdidos, se utilizó el análisis de regresión por pasos. En este se introdujeron como variable dependiente la puntuación estandarizada del promedio de las calificaciones otorgadas a las 4 historias de cada madre y como variables independientes las puntuaciones $\mathrm{Z}$ de las variables edad de la madre, nivel de instrucción, número de hijos y nivel socioeconómico. Asimismo, y dado que en los análisis exploratorios se encontró que las variables nivel de instrucción, número de hijos y nivel socioeconómico se encontraban significativamente asociadas con la edad, se introdujo en la ecuación de la regresión, cuatro variables de interacción (edad con nivel de instrucción, edad con el número de hijos, edad con la edad del hijo menor y edad con el nivel socioeconómico).

Previamente, se verificaron los supuestos básicos para realizar el análisis de regresión: que la variable dependiente se encuentre significativamente asociada a todas las independientes; la independencia de los errores a través del estadístico Durbin Watson $(D B=1.80)$, la independencia de los pronósticos y los residuos tipificados de acuerdo al diagrama de dispersión y la distribución normal de las frecuencia de los residuos estandarizados. Además se encontró que si bien las variables independientes se agrupan en dos factores, el índice de condición muestra un valor adecuado (1). Sin embargo, las variables independientes mostraron cierta colinealidad dado que los valores de tolerancia y de los factores de inflación no son adecuados (1 para ambos casos).

Finalmente, se realizó un análisis de moderación con las variables edad y nivel de instrucción, utilizando el análisis de regresión con el método "Introducir" para calcular los valores de los coeficientes y luego graficar la moderación utilizando el programa Excel. 
Factores sociodemográficos explicativos del guion de base segura materno / Nóblega et al.

\section{Resultados}

La seguridad promedio del guion de base segura de las madres evaluadas fue de 2.18 (IC 95\% = [2.07, 2.29]), DE = .50, Min = 1.1, Max $=4.1$, además más del $50 \%$ de las madres obtuvieron puntuaciones cercanas al promedio siendo muy pocos los valores extremos.

Además, se encontró que el nivel de base segura del guion evidenciadas en los escenarios de la interacción madre-hijo estaban relacionadas $(r=.58, p<.001)$ al igual que las historias de los escenarios de la interacción entre adultos $(r=.45, p<.001)$. Consistentemente con este resultado, las historias de ambos escenarios no difieren significativamente $\left(M_{\text {Historias madre-hijo }}=2.16, D E_{\text {Historias madre-hijo }}=.54, M_{\text {Historias adultos }}\right.$ $\left.=2.08, D E_{\text {Historias adultos }}=.34, t(80)=1.44, p=.16\right) \mathrm{y}$ se encuentran significativamente asociadas $(r=.45, p<.001)$.

En los análisis exploratorios, se encontró que el guion de base segura y la edad de la madre se correlacionan de manera significativa $(r=.41, I C 95 \%=[.28, .58]), p<.001)$. Asimismo, al comparar el nivel de base segura del guion en los diferentes grupos de edad: adolescentes (15 a 19 años), jóvenes (20 a 30 años) y mayores (31 a 45 años) se encontraron diferencias significativas $(F(2)=9.33, p<.001)$. Tal como se observa en la Tabla 1, la comparación por pares, evidencia que madres mayores tienen mayor nivel de base segura que las madres adolescentes y las madres jóvenes, mientras que las madres adolescentes y las madres jóvenes no difieren en su nivel de base segura.

Por otro lado, tal como se observa en la Tabla 2, los análisis preliminares mostraron diferencias significativas en el nivel de base segura del guion de las madres a partir del nivel socioeconómico, el grado de instrucción y el número de hijos. De la misma manera, la correlación del guion con el nivel de instrucción de la madre es significativa $(r=.38, I C 95 \%=[.22, .52]), p<.001)$, así como con el número de hijos $(r=.39$, IC 95\% $=[.18, .59]), p<.001)$ y con el nivel socioeconómico $(r=.40$, IC 95\% $=[.19, .57]), p<.001)$. 


\section{Tabla 1}

Diferencias en el nivel de base segura entre grupos de edad de las madres

\begin{tabular}{|c|c|c|c|c|c|c|}
\hline \multirow[b]{2}{*}{ Edad de la madre } & \multicolumn{2}{|c|}{$\begin{array}{c}\text { Representaciones } \\
\text { de apego }\end{array}$} & \multirow[b]{2}{*}{$\mathrm{t}(81)$} & \multirow[b]{2}{*}{$\mathrm{p}$} & \multirow[b]{2}{*}{$\mathrm{d}$} & \multirow[b]{2}{*}{$1-\beta$} \\
\hline & M & $\mathrm{DE}$ & & & & \\
\hline Adolescentes & 1.98 & .22 & \multirow{2}{*}{-1.45} & \multirow{2}{*}{.15} & \multirow{2}{*}{.40} & \multirow{2}{*}{.45} \\
\hline Jóvenes & 2.10 & .37 & & & & \\
\hline Adolescentes & 1.98 & .22 & \multirow{2}{*}{-3.56} & \multirow{2}{*}{$<.001$} & \multirow{2}{*}{1.05} & \multirow{2}{*}{.97} \\
\hline Mayores & 2.52 & .69 & & & & \\
\hline Jóvenes & 2.10 & .37 & \multirow{2}{*}{-2.64} & \multirow{2}{*}{.01} & \multirow{2}{*}{.71} & \multirow{2}{*}{.83} \\
\hline Mayores & 2.52 & .69 & & & & \\
\hline
\end{tabular}

\section{Tabla 2}

Diferencias en la seguridad del guion de base segura entre grupos de diferentes características sociodemográficas

\begin{tabular}{|c|c|c|c|c|c|c|}
\hline & \multicolumn{2}{|c|}{$\begin{array}{c}\text { Representaciones } \\
\text { de apego }\end{array}$} & \multirow[b]{2}{*}{$\mathrm{t}(81)$} & \multirow[b]{2}{*}{$p$} & \multirow[b]{2}{*}{$d$} & \multirow[b]{2}{*}{$1-\beta$} \\
\hline & M & $\mathrm{DE}$ & & & & \\
\hline \multicolumn{7}{|c|}{ Nivel socioeconómico } \\
\hline Medio & 2.40 & .70 & \multirow{2}{*}{-3.08} & \multirow{2}{*}{.004} & \multirow{2}{*}{.81} & \multirow{2}{*}{.94} \\
\hline Bajo & 2.03 & .23 & & & & \\
\hline \multicolumn{7}{|l|}{ Nivel de instrucción } \\
\hline Secundaria & 2.01 & .27 & \multirow{3}{*}{-3.40} & \multirow{3}{*}{$<.001$} & \multirow{3}{*}{.76} & \multirow{3}{*}{.92} \\
\hline Superior & 2.40 & .63 & & & & \\
\hline Sin pareja & 2.06 & .35 & & & & \\
\hline \multicolumn{7}{|l|}{ Número de hijos } \\
\hline Un hijo & 2.05 & .29 & \multirow{2}{*}{-3.08} & \multirow{2}{*}{.01} & \multirow{2}{*}{.68} & \multirow{2}{*}{.71} \\
\hline Más de un hijo & 2.63 & .77 & & & & \\
\hline
\end{tabular}


El análisis de regresión mostró que el puntaje de base segura del guion de las madres es predicha por la interacción entre la edad de la madre y su nivel de instrucción $(B=.450, \mathrm{EE}=.099$, IC $95 \%=$ $[.252, .647], t=4.533, p<.001)$. La interacción de la edad y el nivel de instrucción explica el $19.2 \%$ de las variaciones en el nivel de base segura del guion de las madres. A pesar de este bajo poder explicativo, se encuentra que este modelo es aceptable $(F=20.547, p<.001)$ con un índice de tamaño de efecto medio $\left(f^{2}=.238\right)$.

Tal como se observa en el Gráfico 1, el análisis de moderación demostró que en el grupo de madres con un mayor nivel de instrucción, el nivel de base segura se incrementa con la edad, mientras que en el grupo de madres de menor nivel de instrucción, el nivel de base segura disminuye conforme se incrementa la edad de la madre.

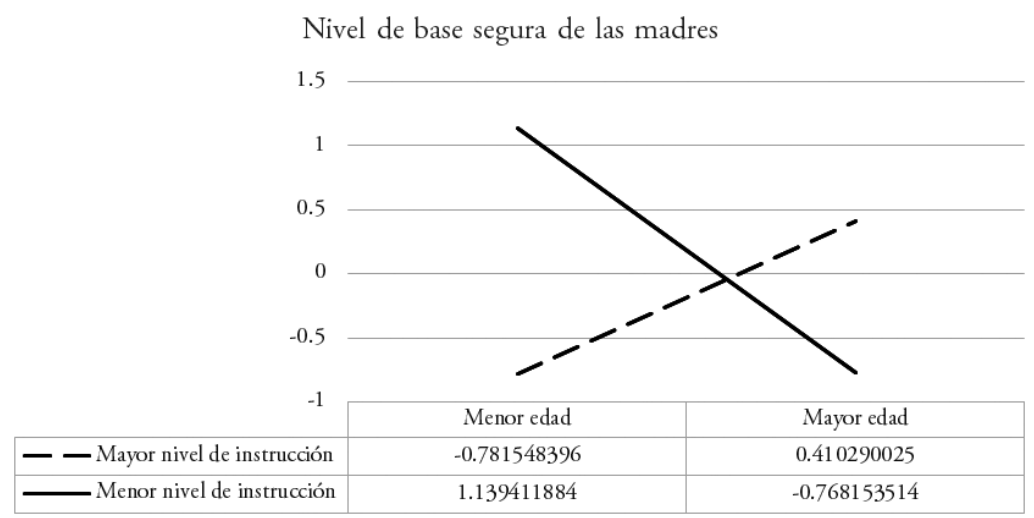

Gráfico 1. Moderación de la variable nivel de instrucción en la relación entre el nivel de base segura de la madre y su edad

\section{Discusión}

El presente estudio tuvo por finalidad principal evaluar el valor explicativo de algunos factores sociodemográficos maternos como la edad, nivel de instrucción, número de hijos y nivel socioeconómico en los guiones de base segura de un grupo de madres. 
Como parte de los resultados descriptivos y en relación al nivel de base segura de los guiones, se encontró que las madres del estudio se caracterizan por presentar menos niveles de base segura comparados con otros estudios (Rodrigues-Doolabh et al., 2001; Tini et al., 2003; Vaughn et al., 2007; Waters \& Rodrigues-Doolabh, 2001) pudiendo ser considerados guiones de apego inseguros. Este bajo nivel de base segura de los guiones es consistente en los distintos escenarios de las narrativas (historias madre-bebé e historias de adultos), dicha consistencia ha sido reportada en anteriores investigaciones (Rodrigues-Doolabh et al., 2001; Tini et al., 2003), lo cual confirma los postulados teóricos en torno al uso de los guiones de base segura en los diferentes escenarios de las interacciones adultas (Bowlby, 1969; Main, 2000; Rholes, Simpson \& Friedman, 2006).

Como hipótesis explicativa de estos resultados, se plantea que la inseguridad presentada en el grupo total de madres podría deberse a aspectos propios de las historias vinculares tempranas de las madres participantes. Al respecto, hay evidencias de que la sensibilidad, tanto materna como paterna, son precedentes importantes para un guion de base segura en la adultez (Hesse \& Main, 2000; Steele et al., 2014; Waters et al., 2013; Waters \& Rodrigues-Doolabh, 1998; Waters \& Cummings, 2000). Estudios previos han demostrado niveles bajos de sensibilidad materna en diversos grupos socioeconómicos del contexto peruano (Nóblega, 2012; Nóblega, Bárrig, Núñez del Prado \& Conde, investigación en curso). Asimismo, las estadísticas muestran que las interacciones tempranas en el contexto peruano están teñidas por experiencias de maltrato físico y psicológico (Instituto Nacional de Estadística e Informática, 2014).

Sin embargo, la ausencia de estudios previos sobre los guiones de base segura en el contexto peruano, no permite una valoración real de la seguridad de los guiones de apego de las madres participantes. Por ello, los hallazgos de este estudio deben considerarse como resultados iniciales que necesitan ser profundizados en posteriores estudios. En esta línea, se considera importante la realización de estudios longitudinales que brinden una mayor explicación de la trayectoria de los guiones de 
base segura desde la niñez hasta la adultez, tanto en contextos de cierta estabilidad socioemocional como en los contextos de mayor adversidad.

Por otro lado, otra hipótesis explicativa debe considerar las características del contexto estudiado. Al respecto, se ha descrito que la expresión o inhibición afectiva tienen importantes variaciones culturales (Hofstede, 1991; Mesquita \& Fridja, 1992), en particular, se considera que en los contextos latinoamericanos, las emociones no son nombradas de manera explícita y se tiende a la evitación de conflictos (Zubieta, Fernández, Vergara, Martínez \& Candia, 1998). En este estudio, la exploración de las representaciones de apego, a partir de la inclusión del fenómeno de la base segura en las narrativas verbales, plantea en primer lugar, la realización de una tarea verbal, en segundo lugar, la calificación de las mismas, exige la expresión de las emociones de forma explícita para alcanzar las puntuaciones más altas. Por lo tanto, en un contexto latinoamericano caracterizado por la escasa verbalización de las emociones es factible que el componente afectivo de las interacciones tendría dificultades para ser verbalizado en forma de narrativas, especialmente frente a un tema que puede resultar angustiante o conflictivo para la persona. Por ello, valdría la pena considerar variables de la psicología cultural como la expresión e inhibición de emociones, la evitación de la incertidumbre para conocer más las características de las madres peruanas y así entender los resultados obtenidos (Fernández, Zubieta \& Páez, 2001).

Adicionalmente, se considera pertinente incorporar en estudio una variable como la longitud de las historias narradas por las madres, ya que en el presente estudio se tuvo historias muy cortas, las cuales, según la literatura, no permiten capturar el fenómeno de base segura (Vaughn et al., 2007).

Por otro lado, los resultados univariados mostraron que las madres de 31 a 45 años tienen los mayores valores del nivel de base segura en sus guiones, aun cuando estos sean de naturaleza insegura, comparadas con las madres jóvenes y las madres adolescentes. Con relación a la diferencia entre las madres de 31 a 45 años y las adolescentes, se podría considerar que el menor nivel de la base segura de los guiones 
de las adolescentes podría deberse al proceso de integración de las figuras de apego que tiene lugar durante esta etapa (Allen \& Land, 1999; van IJzerdoorn \& Bakermans-Kranenburg, 1996) además de otras demandas propias de la etapa evolutiva (Allen, 2008), más aun cuando se atraviesa por la experiencia de la maternidad. Al respecto, se sugiere realizar mayores investigaciones ya que en otros estudios no se ha encontrado diferencias entre la proporción de representaciones seguras e inseguras entre muestras de adolescentes y adultas (van IJzerdoorn \& Bakermans-Kranenburg, 1996). No obstante, es importante considerar que en muchos estudios se utiliza el AAI como medida, lo cual, como se ha explicado, es una vía alterna de acceso a las representaciones de apego a la elegida para este estudio.

Por el contrario, pareciera que el guion de base segura es similar entre las madres adolescentes y las jóvenes (de 20 a 30 ańos). Estos resultados refuerzan hallazgos previos referidos a la ausencia de diferencias entre las madres adolescentes y jóvenes en la interacción con los hijos y en sus representaciones mentales de la maternidad en el contexto peruano (Traverso, 2006). Por otro lado, se debe considerar que la maternidad entre los 20 y 25 ańos, todavía podría ser considerada temprana y por ello este grupo compartiría algunas de las características de la maternidad en adolescentes.

La asociación entre la edad y el nivel de base segura de los guiones de las madres podría evidenciar que las experiencias de interacción se van acumulando con los ańos (Lamb et al., 1985; Sroufe, 2005). Asimismo, la relación entre el nivel de base segura y el nivel de instrucción corrobora los resultados de estudios realizados al respecto (Coppola et al., 2006; Vaughn et al., 2007; Benziesle \& Mychasiuk, 2009; LopezHiges et al., 2013). Aun cuando en este estudio se hayan comprobado hallazgos de investigaciones previas, una hipótesis explicativa de la relación entre el nivel de instrucción y el nivel de base segura podría ser que el mayor nivel de instrucción brinda a las madres una mayor capacidad verbal la cual a su vez permite que las historias muestren de manera más evidente un guion de base segura. Una hipótesis alternativa podría ser que el mayor nivel de instrucción permita una mayor integración de 
las experiencias previas y que esta se evidencie en la base segura de los guiones. Dado que los resultados de este estudio no permiten brindar evidencia a una u otra hipótesis, se considera que es necesaria la realización de estudios adicionales para explicar mejor la relación entre estas variables.

Al considerar estas variables en conjunto, la ecuación de regresión muestra que la interacción de estas dos variables, edad y nivel de instrucción, es importante para explicar el nivel de base segura de los guiones de la madre, mientras que las variables edad, nivel de instrucción, número de hijos y NSE consideradas de forma independiente, no introducen cambios significativos en el nivel de base segura de los guiones.

En primer lugar, el poder explicativo conjunto que tienen la edad y el nivel de instrucción brinda evidencia a favor del dinamismo de estas estructuras mentales (Hamilton, 2000; Weinfield, Sroufe \& Egeland, 2000) a pesar de su estabilidad a lo largo del tiempo (Ainsworth, 1982; Bowlby, 1973; Morales \& Santelices, 2007).

Sin embargo, los resultados de este estudio también muestran que la influencia de la edad sobre el nivel de base segura de los guiones es diferencial de acuerdo al nivel de instrucción de la madre. Así, se evidenciaría que el incremento del nivel de base segura con la edad, solo se da en presencia de mayor nivel de instrucción. Dicho esto, los resultados obtenidos en este estudio corroboran parcialmente la literatura revisada, ya que la experiencia en sucesivas interacciones obtenida con la edad incrementa y nutre los guiones solo para ciertas condiciones.

En este sentido, se podría suponer que un mayor nivel de instrucción estaría siendo un factor que permite a la persona enriquecer el guion de base segura a partir de las distintas experiencias conforme pasan los años. Al parecer en los niveles de instrucción más bajos, estas experiencias tenderían más bien a deteriorar la calidad de los vínculos internalizados en lo que respecta al fenómeno de la base segura. Así, el nivel de instrucción sería un factor protector a medida que pasan los años, estos resultados corroboran la idea de Goez y Smith (2013) quienes consideran que el nivel de instrucción es un factor protector 
para la preservación de las habilidades vinculadas a la teoría de la mente en edades avanzadas.

Estos resultados indicarían que la edad y el nivel de instrucción son las variables sociodemográficas que mejor predicen el nivel de base segura en los guiones. Sin embargo, hay que considerar que la edad y el nivel de instrucción estuvieron alta y directamente asociada a las otras variables estudiadas. Esta alta correlación entre las variables consideradas, no permite descartar que en otras muestras más grandes, las otras variables también tengan un poder explicativo del nivel de base segura de los guiones. Asimismo, y aun siendo un modelo adecuado, es probable que otras variables, no incluidas en este estudio, también puedan explicar el fenómeno de la base segura en los guiones.

Otra limitación de los resultados obtenidos en esta investigación, y en relación con lo señalado anteriormente, es que utiliza dos grupos de participantes que fueron evaluados en dos momentos diferentes, lo cual podría hacer que no se haya controlado el rol de otras variables importantes que podrían estar influyendo en los resultados obtenidos. Esta limitación cobra mayor importancia al comprobar que las madres de uno de los grupos tienen menor edad, menor nivel de instrucción, menos hijos y sus hijos son menores. Considerando esta limitación, los resultados encontrados en la presente investigación no son conclusivos sino más bien un punto de partida para continuar realizando estudios en esta temática.

A pesar de estas limitaciones, este estudia aporta a la comprensión de un tema aun poco abordado en especial en los contextos latinoamericanos en los que prima la diversidad de las condiciones sociodemográficas de las madres y las diferentes repercusiones que tienen estas sobre las características de la conducta materna. 
Factores sociodemográficos explicativos del guion de base segura materno / Nóblega et al.

\section{Referencias}

Adler, N., Boyce, T., Chesney, M., Cohen, S., Folkman, S., Kahn, R. \& Syme, S. L. (1994). Socioeconomic Status and Health. American Psychologist. 49(1), 15-24. https://doi.org/10.1037/0003-066X. 49.1.15

Ainsworth, M. (1982). Attachment: Retrospect and prospect. En C. M. Parkes \& J. Stevenson-Hinde (Eds.), The place of attachment in human behavior, (pp. 3-30). New York: Basic Books.

Allen, J. (2008). The attachment system in adolescence. En J. Cassidy y P. R. Shaver (Eds.), Handbook of attachment: Theory, research and clinical applications (2da ed., pp. 419-435). New York: The Guilford Press.

Allen, J. \& Land, D. (1999). Attachment in Adolescence. En J. Cassidy y P. R. Shaver (Eds.), Handbook of attachment: Theory, research and clinical applications (pp. 319-335). New York: The Guilford Press.

Allen, J., Boykin, K., Kuperminc, G. \& Jodl, K. (2004). Stability and change in attachment security across adolescence. Child Development,75(6),1792-1805.https://doi.org/10.1111/j.14678624.2004.00817.x

Anafarta, M. (2007). The psychometric qualities of the knowledge of secure base script-prompt word outline method: multi-sample evaluation of the validity and the reliability (Tesis doctoral). Graduate School of Social Sciences of Middle East Technical University.

Bakermans-Kranenburg, M. (2006). Script-like attachment representations: Steps towards a secure base for further research. Attachment \& Human Development, 8, 275-281. https://doi. org/10.1080/14616730600910037

Belsky, J. (1984). The determinants of parenting: A process model. Child Development, 55(1), 83-96. https://doi.org/10.2307/1129836

Benziesle, K. \& Mychasiuk, R. (2009). Fostering family resiliency: a review of the key protective factors. Child and Family Social Work, 14, 103-114. https://doi.org/10.1111/j.13652206.2008.00586.x 
Bost, K., Shin, N., Mcbride, B., Brown, G., Vaughn, B., Coppola, G., Veríssimo, M., Monteiro, L. \& Korth, B. (2006). Maternal secure base scripts, children's attachment security, and mother child narrative styles. Attachment \& Human Development, 8(3), 241-260. https://doi.org/10.1080/14616730600856131

Bowlby, J. (1969). Attachment and loss (1): Attachment. New York: Basic Books.

Bowlby, J. (1973). Attachment and loss (2): Separation, anxiety, and anger. New York: Basic Books.

Bretherton, I. (1990). Communication patterns, internal working models, and the Intergenerational transmission of attachment relationship. Infant Mental Health Journal, 11, 237-252. https://doi.org/10.1002/1097-0355(199023)11:3<237::AIDIMHJ2280110306>3.0.CO;2-X

Bretherton, I. \& Munholland, K. (2008). Internal working models in attachment relationships: Elaborating a central construct in attachment theory. En J. Cassidy \& P. R. Shaver (Eds.), Handbook of attachment: Theory, research, and clinical applications (2da ed., pp. 102-127). New York: Guilford Press.

Bretherton, I., Ridgeway, D. \& Cassidy, J. (1990). Assessing Internal Working Models of the Attachment Relationship: An Attachment Story Completion Task for 3-Year-Olds. En M. T. Greenberg, D. Cicchetti, y E. M. Cummings (Eds.), Attachment in the Preschool Years (pp. 273-308). Chicago: University of Chicago Press.

Chalasani, Kelly, Mensch \& Soler-Hampejsek (agosto, 2013). Adolescent pregnancy and education trajectories in Malawi. Paper presentado en la XXVII IUSSP International Population Conference, Busan, República de Korea. Recuperado de http:// paa2013.princeton.edu/papers/132776.

Coleman, P. \& Karraker, K. (2000). Parenting self-efficacy among mothers of school-age children: Conceptualization, measurement, and correlates. Family Relations, 49, 13-24. https://doi.org/10.1111/j.1741-3729.2000.00013.x 
Factores sociodemográficos explicativos del guion de base segura materno / Nóblega et al.

Coppola, G., Vaughn, B., Cassibba, R. \& Costantini, A. (2006). The attachment script representation procedure in an Italian sample: Associations with adult attachment Interview scales and with maternal sensitivity, Attachment \& Human Development, 8(3), 209-219. https://doi.org/10.1080/14616730600856065

Crowell, J., Treboux, D. \& Waters, E. (2002). Comparing Adult Attachment Measures: Relations to Attachment Behavior and Feelings. Poster presented en el American Academy of Child and Adolescent Psychiatry, San Francisco: California.

Crowell, J. A \& Waters, E. (2005). Attachment representations, secure base behavior, and the evolution of adult relationships: The Stony Brook Adult Relationship Project. En K. E. Grossmann, K. Grossmann \& E. Waters (Eds.), Attachment from infancy to adulthood: The major longitudinal studies (pp. 223-244). New York: The Guilford Press.

De Falco, S., Emer, A., Martini, L., Rigo, P., Pruner, S. \& Venuti, P. (2014). Predictors of mother-child interaction quality and child attachment security in at-risk families. Frontiers in Psychology. 1(5), 29-38.

Dykas, M., Woodhouse, S., Cassidy, J. \& Waters, H. (2006). Narrative assessment of attachment representations: Links between secure base scripts and adolescent attachment. Attachment \& Human Development, 8, 221-240. https://doi. org/10.1080/14616730600856099

Faul, F., Erdfelder, E., Buchner, A. \& Lang, A. (2009). Statistical power analyses using $\mathrm{G}^{*}$ Power 3.1: Tests for correlation and regression analyses. Behavior Research Methods, 41, 1149-1160. https://doi. org/10.3758/BRM.41.4.1149

Fernández, I., Zubieta, E. \& Páez, D. (2001). Emocionarse en Latinoamérica. International Journal of Social Sciences and Humnanities (Sociotam), 11(1), 29-53.

Fivush,R.,(2006).Scriptingattachment:Generalizedeventrepresentations and internal working models. Attachment and Human Behavior, 8, 283-289. https://doi.org/10.1080/08912960600858935 
Goez, M \& Smith, P. (2013). Theory of mind, old age and educational opportunity in Colombia. The international journal of aging and human development, 77(2), 107-125. https://doi.org/10.2190/ AG.77.2.b

Grossmann, K., Grossmann, K. \& Kindler, H. (2005). Early care and the roots of attachment and partnership representations in the Bielefeld and Regensburg Longitudinal Studies. En K. E. Grossmann, K. Grossmann \& E. Waters (Eds.), Attachment from infancy to adulthood: The major longitudinal studies (pp. 98-136). New York: The Guilford Press.

Guttmann-Steinmetz, S., Elliot, M., Steiner, M. \& Waters, H. (2003). Co-constructing script-like representations of early secure base experience. Poster presentado en The biennial meeting of the Society for Research in Child Development. Tampa: Florida

Hamilton, C. (2000). Continuity and discontinuity of attachment from Infancy through Adolescence. Child Development, 3(7), 690-694. https://doi.org/10.1111/1467-8624.00177

Hazan, C. \& Shaver, P. (1987). Romantic Love conceptualized as an attachment process. Journal of Personality and Social Psychology, 52(3), 511-524. https://doi.org/10.1177/00030651000480041101

Hesse, E. \& Main, M. (2000). Disorganized infant, child and adult attachment: Collapse in behavioral and attentional strategies. Journal of the American Psychoanalytic Association, 48(4), 10971127.

Hofstede, G. (1991). Cultures and Organizations: Software of the Mind. Londres: McGraw-Hill.

Huth-Bocks, A. (2014). Secure base scripts are associated with maternal parenting behavior across contexts and reflective functioning among trauma-exposed mothers. Attachment Human \& Development, 16(6), 535-556. https://doi.org/10.1080/14616734.2 014.967787

IBM, Inc. (2010). SPSS Statistics. Version 19.0. Armonk, NY: IBM Corp. 
Factores sociodemográficos explicativos del guion de base segura materno / Nóblega et al.

Instituto Nacional de Estadística e Informática - INEI (2014). Encuesta Demográfica y de Salud Familiar. Lima: Instituto Nacional de Estadística e Informática.

Kapp, M. (1998). Mothers' perceptions of confidence with self-care and infant care. Journal of Perinatal Education, 7, 17-25. https:// doi.org/10.1624/105812498X86864

Lamb, M., Thompson, R., Gardner, W. \& Charnov, E. (1985). Infantmother attachment: The origins and significance of individual differences in Strange Situation behavior. Hillsdale: Erlbaum.

López-Higes, R., Rubio-Valdehita, S., Prados, J. \& Galindo, M. (2013) Reserva cognitiva y habilidades lingüísticas en mayores sanos. Revista Neurológica, 57, 97-102.

Main, M. (2000). The organized categories of infant, child, and adult attachment: Flexible vs. inflexible attention under attachmentrelated stress. Journal of the American Psychoanalytic Association, 48, 1055-1096. https://doi.org/10.1177/00030651000480041801

Main, M., Kaplan, N. y Cassidy, J. (1985). Security in Infancy, Childhood, and Adulthood: a Move to the Level of Representation. Monographs of the Society for Research in Child Development, 50(2), 66-104. https://doi.org/10.2307/3333827

Marrone, M. (2001). La teoría del apego: un enfoque actual. Madrid: Psimática.

Mesquita, B. \& Fridja, N. (1992). Cultural variations in emotions: A review. Psychological Bulletin, 112, 179-20. https://doi.org/ 10.1037/0033-2909.112.2.179

Mikulincer,M \& Shaver, P. (2007) Boosting attachment security to promote mental health, prosocial values, and inter-group tolerance. Psychological Inquiry, 18, 139-156. https:/doi.org/ 10.1080/10478400701512646

Monteiro, L., Veríssimo, M., Vaughn, B., Santos, A. \& Bost, K. (2008). Secure base representations for both fathers and mothers predict children's secure base behavior in a sample of Portuguese families. Attachment and Human Development, 10, 189-206. https://doi.org/10.1080/14616730802113711 
Morales, S. \& Santelices, M. (2007). Los modelos operantes internos y sus abordajes en psicoterapia [Working Models in Adult Attachment and Psychotherapy Interventions]. Revista Terapia Psicológica, 25, 163-172. https://doi.org/10.4067/s071848082007000200007

Nóblega, M. (2012). Conducta de base segura y sensitividad en niños y madres del distrito de los Olivos. Tesis para optar por el grado de Doctora en Psicología. Lima: Pontificia Universidad Católica del Perú.

Nóblega, M., Bárrig, P., Núñez del Prado, J. \& Conde, G. (investigación en curso). Regulación de emociones de niños pre-escolares: Estudio longitudinal de su relación con los aspectos representacionales y comportamentales del apego del niño y de la conducta materna.

Pelchat, D., Bisson, J., Bois, C. \& Saucier, J. (2003). The effects of early relational antecedents and other factors on the parental sensitivity of mothers and fathers. Infant Child Development, 12, 27-51. https://doi.org/10.1002/icd.335

Pinedo, J. \& Santelices, M. (2006). Apego Adulto: Los Modelos Operantes Internos y la Teoría de la Mente [Internal Working Models and the Theory of Mind]. Terapia Psicológica, 24(2), 201-210.

Raval, V., Goldberg, S., Atkinson, L., Benoit, D., Myhal, N., Poulton, L. \& Zwiers, M. (2001). Maternal attachment, maternal responsiveness and infant attachment. Infant Behavior \& Development, 24, 281-304. https://doi.org/10.1016/S01636383(01)00082-0

Rholes, S., Simpson, J. \& Friedman, M. (2006). Avoidant attachment and the experience of parenting. Personality and social psychology bulletin, 3(32). https://doi.org/10.1177/0146167205280910

Rodrigues-Doolabh, L., Wais, D., Zevallos, A. \& Rodrigues, R. (Abril, 2001). Attachment scripts across cultures. Paper presentado en el Biennial Meetings of the Society for Research in Child Development, Minneapolis. Recuperado de http://www. 
psychology.sunysb.edu/attachment/srcd2001/CrossCulture/ LR-SRCD2110.pdf.

Rodrigues-Doolabh, L., Zevallos, A., Turan, B. \& Green, J. (Abril, 2003). Attachment scripts across cultures. Paper presentado en el Biennial Meetings of the Society for Research in Child Development, Tampa, Florida. Recuperado de http://www. psychology.sunysb.edu/attachment/srcd2003/srcd2003.htm.

Sánchez, J. \& Hidalgo, M. (2002). Madres adultas y madres adolescentes. Un análisis comparativo de las interacciones que mantienen con sus bebés. Apuntes de Psicología, 20(2), 243-256.

Schoenmaker, C., Juffer, F., van Ijzendoorn, M. H., Linting, M., van der Voort, A. \& Bakermans-Kranenburg, M. (2015). From maternal sensitivity in infancy to adult attachment representations: A longitudinal adoption study with secure base scripts. Attachment \& Human Development, 17(3), 241-256. https://doi.org/10.1080/14616734.2015.1037315

Simpson, J., Collins, W., Tran, S. \& Haydon K. (2007). Attachment and the experience and expression of emotions in adult romantic relationships: A developmental perspective. Journal of Personality and Social Psychology, 92(1), 355-367. https://doi. org/10.1037/0022-3514.92.2.355

Sroufe, L. (2005). Attachment and development: A prospective, longitudinal study from birth to adulthood. Attachment \& Human Development, 7(4), 349-367. https://doi. org/10.1080/14616730500365928

Steele, R., Waters, T., Vaughn, B., Truitt, W., Booth-LaForce, C., Bost, K., Waters, H. \& Roismn, G. (2014). Caregiving antecedents of secure base script knowledge: A comparative analysis of young adult attachment representations. Developmental psychology, 11(50), 2526-2538. https://doi.org/10.1037/a0037992

Tini, M., Corcoran, D., Rodrigues-Doolabh, L. \& Waters, E. (Marzo, 2003). Maternal attachment scripts and infant secure base behavior. En H. Waters \& E. Waters (Orgs.), Script-like representations of secure base experience: Evidence of cross-cultural, 
and behavioral links. Simposio presentado en el Biennial Meetings of the Society for Research in Child Development, Tampa, Florida. Recuperado de http://www.psychology.sunysb. edu/attachment/srcd2003/srcd2003.htm.

Traverso, P. (2006). Adolescent mothers in a context of poverty in Perú: Maternal representations and mother-infant interactions (Tesis doctoral). Katholieke Universiteit Leuven, Bélgica.

van IJzendoorn, M. \& Bakermans-Kranenburg, M. (1996). Attachment Representations in mothers, fathers, adolescents, and clinical groups: A Meta-Analytic search for normative data. Journal of Consulting and Clinical Psychology, 1(64), 8-21. https://doi. org/10.1037/0022-006X.64.1.8

van IJzendoorn, M. (1995). Adult attachment representations, parental responsiveness, and infant attachment: A metaanalysis on the predictive validity of the Adult Attachment Interview. Psychological Bulletin, 117, 387-403. https://doi. org/10.1037/0033-2909.117.3.387

Vaughn, B., Coppola, G., Verissimo, M., Monteiro, L., Santos, A., Posada, G., Carbonell, O., Plata, S., Waters, H., Bost, K., McBride, B., Shin, N. \& Korth, B. (2007). The quality of maternal secure-base scripts predicts children's secure-base behavior at home in three sociocultural groups. International Journal of Behavioral Development, 31(1), 65-76. https://doi. org/10.1177/0165025407073574

Vaughn, B., Veríssimo, M., Coppola, G., Bost, K., Shin, N., McBride, B., Krzysik, L. \& Korth, B. (2006). Maternal attachment script representations: Longitudinal stability and associations with stylistic features of maternal narratives. Attachment \& Human Development, 8, 199-208. https://doi. org/10.1080/14616730600856024

Verissimo, M. \& Salvaterra, F. (2006). Maternal secure-base scripts and children's attachment security in an adopted sample. Attachment \& Human Development, 8(3), 261-273. https://doi. org/10.1080/14616730600856149 
von der Lippe, A., Eilertsen, D., Hartmann, E. \& Killèn, K. (2010). The role of maternal attachment in children's attachment and cognitive executive functioning: A preliminary study. Attachment \& Human Development, 12(5), 429-444. https://doi.org/10.108 $0 / 14616734.2010 .501967$

Wais, D. \& Treboux, D. (2003). Current relationship scripts. Paper presentado en the Biennial Meetings of the Society for Research in Child Development, Tampa: Florida.

Waters, E. \& Cummings, E. M. (2000). A Secure Base from which to Explore Close Relationships. Child Development, 71, 164-172. https://doi.org/10.1111/1467-8624.00130

Waters, H., Rodrigues, L. \& Ridgeway, D. (1998). Cognitive underpinnings o narrative attachment assessment. Journal of Experimental Child Psychology, 71, 211-234. https://doi. org/10.1006/jecp.1998.2473

Waters, T., Brockmeyer, S. \& Crowell, J. (2013). AAI coherence predicts caregiving and care seeking behavior: Secure base script knowledge helps explain why, Attachment \& Human Development, 15(3), 316-331. https://doi.org/10.1080/146167 34.2013.782657

Waters, T., Groh, A., Vaughm, B., Veríssimo, M., Fraley, R., Steele, R., Bost, K. \& Coppola, G. (2015) The latent structure of secure base script knowledge. Developmental Psychology, 6(51), 823-830. https://doi.org/10.1037/dev0000012

Waters, H. \& Rodrigues-Doolabh (1998). Narrative assessment of adult attachment representations: the scoring of secure base script content. Stony Brook: New York.

Waters, H. \& Rodrigues-Doolabh, L. (Abril, 2001). Are attachment scripts the building blocks of attachment representations? Narrative assessment of representations and the AAI. Poster presentado en el Simposio What does the Adult Attachment Interview measure and when does it matter? Longitudinal studies of attachment representations en el Biennial Meeting of the Society for Research in Child Development, Minneapolis, Minnesota. 
Recuperado de http://www.psychology.sunysb.edu/attachment/ $\operatorname{srcd} 2001 / \operatorname{srcd} 2001 . h t m$

Waters, H. \& Waters, E. (2006). The attachment working models concept: Among other things, we build script-like representations of secure base experiences. Attachment \& Human Development, 8(3), 185-197. https://doi.org/10.1080/14616730600856016

Weinfield, N., Sroufe, A. \& Egeland, B. (2000). Attachment from Infancy to Early Adulthood in a high risk sample: Continuity, Discontinuity and their correlates. Child Development, 3(71), 695-702. https://doi.org/10.1111/1467-8624.00178

Wong, M., Bost, K., Shin, N., Veríssimo, M., Maia, J., Monteiro, L., Silva, F., Coppola, G., Constantini, A. \& Vaughn, B. (2011). Preschool children's mental representations of attachment: antecedents in their secure base behaviors and maternal attachment scripts. Attachment \& Human Development, 13(5), 489-502. https://doi.org/10.1080/14616734.2011.602256

Zubieta, E., Fernández, I., Vergara, A., Martínez, M. \& Candia, L. (1998). Cultura y emoción en América. Boletín de Psicología, $61,65-90$.

Recibido: 26 de mayo, 2016 Revisado: 17 de febrero, 2017 Aceptado: 23 de febrero, 2017 